\title{
Regeneraciones artificiales en playas heterogéneas: análisis de datos y modelado numérico
}

\author{
Cristóbal Rodríguez Delgado*, \\ Tutores: Dr. Miguel Ortega Sánchez y Rafael J. Bergillos Meca** \\ Grupo de Dinámica de Flujos Ambientales, Instituto Interuniversitario de Investigación del Sistema Tierra en Andalucía (IISTA), Universidad de \\ Granada. Edificio CEAMA, Avda. del Mediterráneo, s/n, 18006, Granada, España. \\ Máster Oficial Interuniversitario en Hidráulica Ambiental. Especialidad: Gestión Integral de Puertos y Costas. Curso: $2015-2016$.
}

\begin{abstract}
Resumen
Las regeneraciones en sistemas deltaicos, mediante el aporte artificial de sedimentos, son cada vez más frecuentes debido a las acciones humanas que desencadenan el retroceso de la línea de costa. Este Trabajo Fin de Máster estudia la evolución de una regeneración realizada en una playa heterogénea situada en el Delta del Guadalfeo (Sur de España), durante los seis meses siguientes a la actuación. Para ello, se han analizado datos de clima marítimo y medidas batimétricas y topográficas. Además, se ha aplicado un modelo de propagación del oleaje; diferentes formulaciones para calcular las tasas de transporte longitudinal de sedimentos (CERC, Inman, Kamphuis y Van Rijn); y un modelo de una línea para reproducir los cambios en la posición de la línea de costa. Los resultados muestran que el transporte longitudinal de sedimentos generado por temporales con dirección oeste-suroeste genera erosión en el tramo de costa estudiado, mientras que los eventos de procedencia este-sureste tienden a recuperarlo. La fórmula que mejor ajusta las medidas realizadas es la de Van Rijn usando como tamaño de grano el de la fracción más gruesa de las predominantes en la playa. Además, el modelo de una línea reproduce mejor el comportamiento de la costa cuanto mayor es el tiempo desde el momento en que se llevó a cabo la regeneración artificial. Se concluye que la bidireccionalidad del oleaje junto con la morfología en planta caracterizan el comportamiento de la costa, siendo la fracción de sedimento más gruesa la de mayor importancia en la respuesta del sistema.
\end{abstract}

Palabras clave: Delta, playa heterogénea, regeneración, modelo propagación de oleaje, transporte longitudinal de sedimentos

\section{Introducción}

Las playas heterogéneas presentan un amplio rango de tamaños de sedimento, con una estratificación vertical cambiante, y cuya proporción y gradación son variables tanto transversal como longitudinalmente. Son playas comunes en costas de todo el mundo, incluyendo buena parte de las playas mediterráneas, británicas, neozelandesas y canadienses, entre otras. Además, la utilización de gravas en la regeneración de playas de arena hace que sean cada vez más frecuentes. A pesar de ello, numerosos estudios destacan la discrepancia entre los progresos hechos en playas de arena y la ausencia

\footnotetext{
*cristobalrodel@gmail.com

**miguelos@ugr.es

rbergillos@ugr.es
}

de avances similares en playas heterogéneas y de grava (Jennings and Shulmeister, 2002; Buscombe and Masselink, 2006).

Por su parte, los deltas se sitúan en la confluencia entre ambientes fluviales y marítimos por lo que son sistemas de gran valor ambiental, social y económico en los que los procesos climáticos y sedimentarios interaccionan de manera compleja. Además, cada vez son más frecuentes las acciones humanas que limitan el aporte de sedimentos por parte del río al delta, como la construcción de presas (Syvitski et al., 2009). Esto hace que los deltas a lo largo de todo el mundo estén sufriendo problemas de erosión y retroceso de la línea de costa (El Banna and Frihy, 2009; Simeoni and Corbau, 2009), que podrán verse incrementados por el previsible ascenso del nivel del mar (IPCC, 2001).

Para intentar paliar estas afecciones, cada vez son 


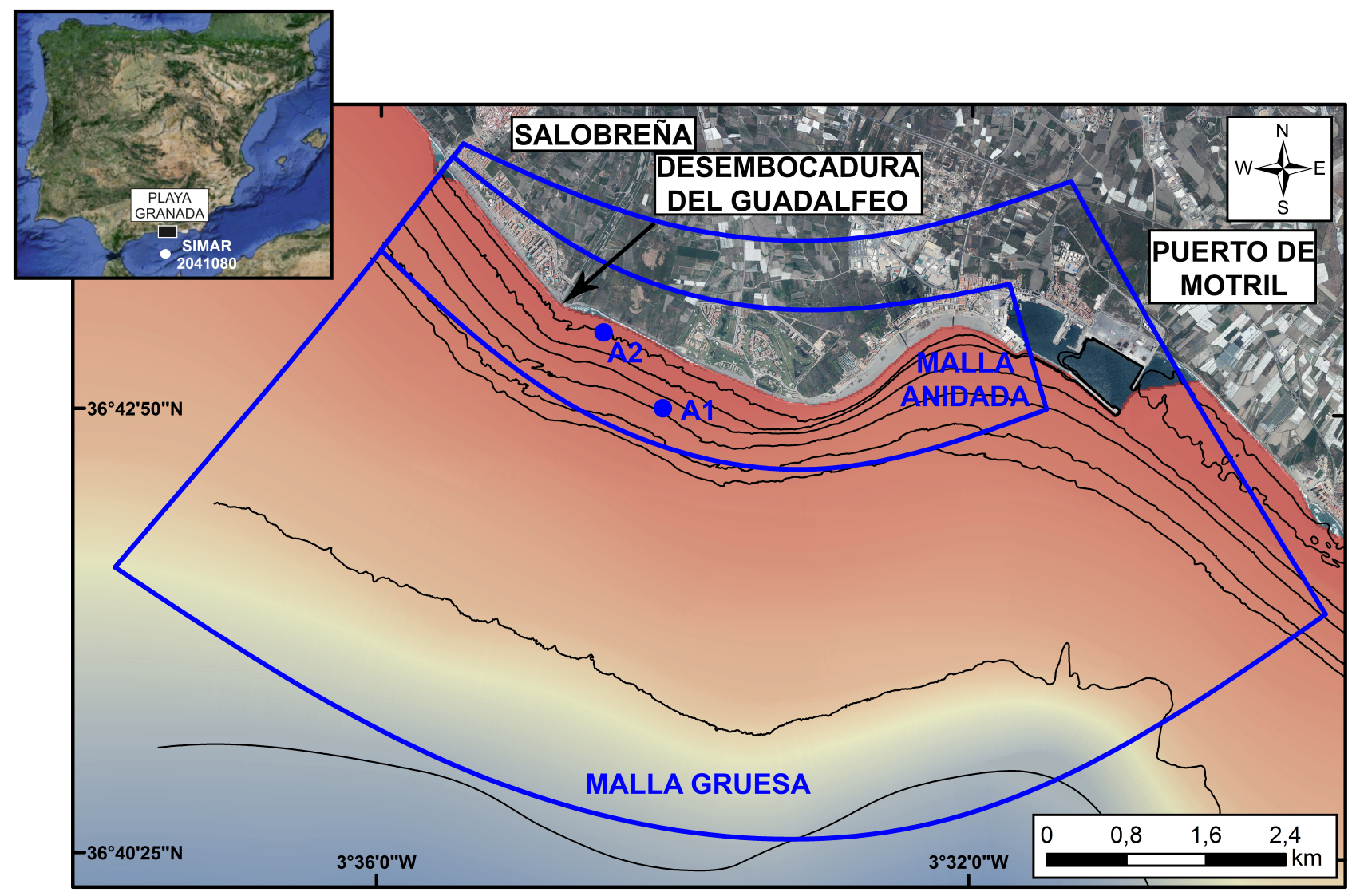

Figura 1: Panel superior izquierdo: Península Ibérica, situación de Playa Granada y el punto SIMAR 2041080. Panel Central: Mallas utilizadas en el modelo de propagación de oleaje, posición de los instrumentos de medida y lugares más importantes de la zona de estudio.

más frecuentes las regeneraciones de playas mediante aportes artificiales de sedimento. Por tanto, parece necesario el estudio de la evolución de estas regeneraciones con el objetivo de ampliar el conocimiento sobre el comportamiento de playas situadas en entornos deltaicos. El seguimiento de estas actuaciones mediante medidas de campo es fundamental para conocer en profundidad como afectan a los procesos que tienen lugar en la costa (de Schipper et al., 2016; Bertoni and Sarti, 2011).

A su vez, los modelos numéricos que predicen el transporte longitudinal de sedimentos y la posición de la línea de costa son una herramienta esencial en el diseño de este tipo de actuaciones (Ávila, 2007; LópezRuiz et al., 2014). Es por eso necesario combinar estos modelos con medidas de campo si se quiere profundizar en el conocimiento del comportamiento de las regeneraciones realizadas en nuestras costas.

Por tanto, el objetivo de este Trabajo Fin de Máster (TFM) es analizar las medidas de campo realizadas tras una regeneración artificial de sedimentos en Playa Granada (Motril) y contrastarlas con los resultados obteni- dos mediante la combinación de un modelo de propagación de oleaje (el Delft3D-Wave), distintas fórmulas de transporte longitudinal de sedimentos (Inman and Bagnold, 1963; USACE, 1984; Kamphuis, 1991; Van Rijn, 2014) y el modelo de una línea.

La estructura de este trabajo es la siguiente: en los apartados 2 y 3 se describen la zona de estudio y la metodología seguida para alcanzar los objetivos propuestos, respectivamente. El apartado 4 detalla los resultados obtenidos: descripción del clima marítimo, propagación del oleaje mediante el modelo Delft3D, evolución del perfil de playa y de la línea de costa, tasas de transporte longitudinal de sedimentos y modelo de una línea; así como su discusión. El apartado 5 expone las principales conclusiones y, por último, en el apartado 6 se incluyen algunas líneas de investigación futura posibles.

\section{Zona de estudio}

Playa Granada es una playa de $3 \mathrm{~km}$ de longitud situada en el sur de la Península Ibérica, en la costa del 
Mar de Alborán (Figura 1). Esta sección de costa se encuentra en el centro de la llanura deltaica del Guadalfeo, limitando en el oeste con la actual desembocadura del mismo y en el este con la Punta del Santo, antigua desembocadura del río. La llanura deltaica del Guadalfeo se encuentra limitada a su vez por el oeste por el Peñón de Salobreña y por el este por el Puerto de Motril, constituyendo este último una barrera artificial que impide el transporte longitudinal de sedimentos en la dirección este (Félix et al., 2012).

El litoral andaluz del mar de Alborán se caracteriza por la presencia de la cordillera Penibética que hace que los cauces fluviales sean cortos y con elevadas pendientes. La fuente más importante de sedimentos en Playa Granada es el río Guadalfeo (Polo et al., 2013). Su cuenca tiene un área de $1252 \mathrm{~km}^{2}$, incluyendo el pico más alto de la Península Ibérica (Herrero et al., 2011), el Mulhacén ( $3400 \mathrm{~m})$. Las precipitaciones son escasas, con una distribución espacial irregular (Moreno Aranda et al., 2010), además, la fuerte pendiente del cauce hace que sea uno de los hidrológicamente más energéticos del litoral mediterráneo y que los sedimentos aportados comprendan un amplio rango de tamaños (Millares et al., 2014). Consecuentemente, Playa Granada es una playa heterogénea con tamaños de grano que varían entre arenas y gravas. De acuerdo con Bergillos et al. (2016), en Playa Granada se identifican tres fracciones predominantes en cuanto al tamaño de sedimento: una de arena $(\sim 0,35 \mathrm{~mm}), \mathrm{y}$ dos de grava $(\sim 5 \mathrm{~mm}$ y $\sim 20$ $\mathrm{mm})$.

Desde el año 2004, la presa de Rules regula el $85 \%$ del caudal del río Guadalfeo (Losada et al., 2011). Con una capacidad total de $117 \mathrm{hm}^{3}$, los usos previstos para el agua del embalse eran: $40 \%$ riego, $30 \%$ control de avenidas, $19 \%$ abastecimiento, $9 \%$ generación de energía y $2 \%$ mantenimiento del caudal ambiental. Sin embargo, la presa actúa como una barrera para el transporte fluvial de sedimentos lo que supone que, desde su entrada en funcionamiento, la costa de Playa Granada sufra importantes problemas de erosión. Las parcelas anexas al tramo de costa en el que se centra este estudio tienen un uso principalmente turístico. En ellas se encuentran un hotel, un campo de golf, restaurantes y apartamentos vacacionales (Félix et al., 2012), que han sido afectados por el fuerte retroceso de la línea de costa en los últimos años (Rodríguez-Delgado, 2015). Por ello, desde la entrada en funcionamiento de la presa en 2004 se han llevado a cabo numerosos proyectos de regeneración artificial en este tramo de costa.

El clima en la zona de estudio sufre un gran contraste entre verano e invierno. Durante el invierno, esta región sufre temporales con vientos de 18 y $22 \mathrm{~m} / \mathrm{s}$, con un fetch de unos 300 km (Ortega-Sánchez et al., 2008). El oleaje es bidireccional dominando las direcciones oestesuroeste y este-sureste. Durante los eventos de tormenta, la altura de ola en profundidades indefinidas supera los $3 \mathrm{~m}$, pudiendo alcanzar picos de 4.5-5 $\mathrm{m}$. El rango de la marea astronómica se sitúa entorno a los $0.6 \mathrm{~m}$, siendo por tanto una zona micromareal (Ortega-Sánchez et al., 2014).

\section{Metodología}

Este Trabajo Fin de Máster se basa en: (1) el análisis de datos de clima y medidas batimétricas y topográficas, y (2) la aplicación de un modelo numérico de propagación de oleaje, diferentes formulaciones para calcular las tasas de transporte longitudinal de sedimentos y un modelo de una línea para reproducir los cambios en la posición de la línea de costa (Figura 2).

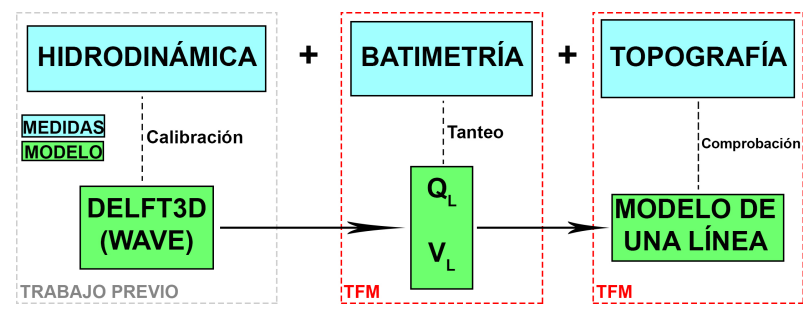

Figura 2: Esquema representativo de la metodología seguida para la realización de este TFM.

\subsection{Datos}

\subsubsection{Regeneración}

La regeneración artificial consistió en el aporte de un volumen de $106,676 \mathrm{~m}^{3}$ de sedimento, con una distribución de tamaño uniforme $\left(D_{5} 0=2 \mathrm{~mm}\right)$, sobre una longitud de $627 \mathrm{~m}$ de costa al sureste de la desembocadura del río Guadalfeo (Figura 3). Los datos relativos a la regeneración fueron proporcionados por el Servicio Provincial de Costas de Granada. Las medidas de campo comenzaron el 23 de diciembre de 2014 (justo después de la regeneración) y acabaron el 21 de junio de 2015, siendo este el periodo de estudio de este TFM.

\subsubsection{Clima}

El análisis del clima marítimo en la zona de estudio durante este periodo se realizó partir de los datos del punto SIMAR 2041080, proporcionados por Puertos del Estado. Se estudió la serie temporal de altura de ola significante en profundidades indefinidas $\left(H_{0}\right)$, identificando la ocurrencia de temporales mediante el método de picos sobre umbral, considerando como valor umbral 

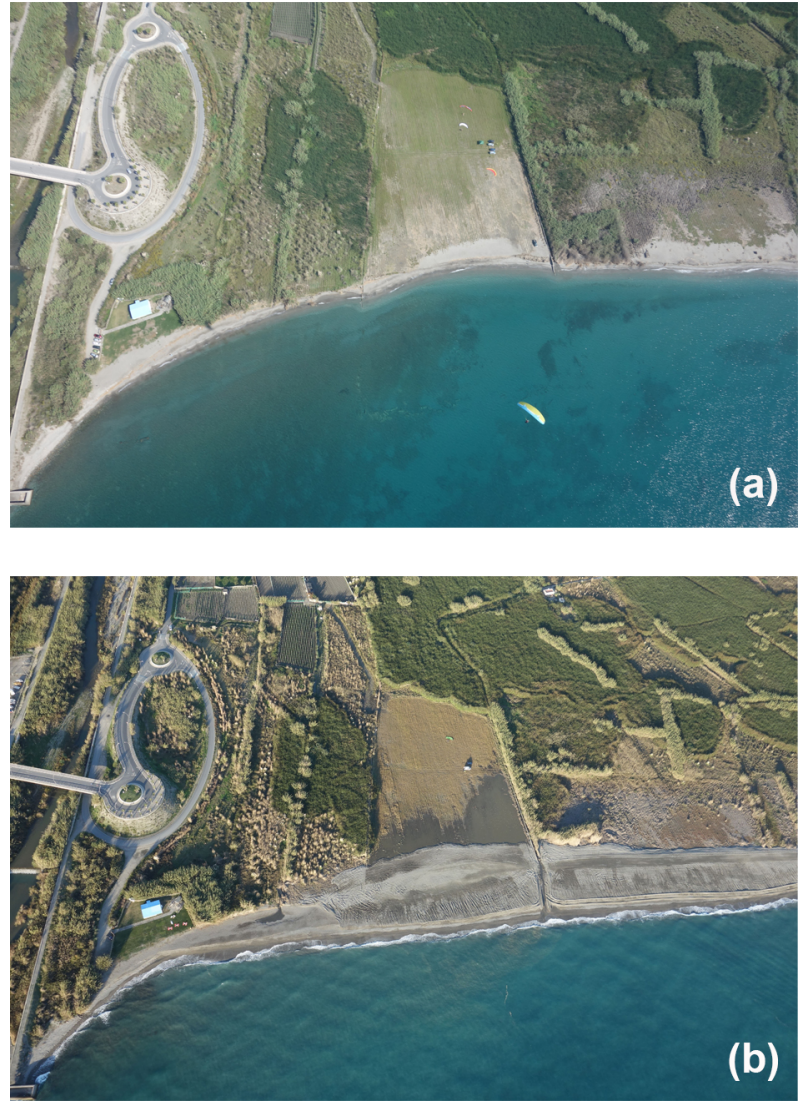

Figura 3: Fotografías de la zona de estudio: (a) antes de la regeneración y (b) después de la regeneración.

$3.1 \mathrm{~m}\left(H_{99} \%\right)$. Además de la altura de ola, también se estudiaron los datos de periodo de pico $\left(T_{p}\right)$, dirección del oleaje y velocidad y dirección del viento.

Los datos de elevación total de la superficie libre han sido obtenidos sumando a los datos de marea astronómica y meteorológica del mareógrafo del Puerto de Motril el run-up causado por el oleaje. Este ha sido calculado mediante la siguiente expresión (Nielsen and Hanslow, 1991):

$$
\Delta \eta_{\text {wave }}=0,36 g^{0,5} H_{0}^{0,5} T_{p} \tan \beta
$$

donde $\tan \beta$ la pendiente en la zona de rompientes y $g$ es la aceleración de la gravedad. Esto se ha usado para aplicar el criterio de umbral utilizado en Bergillos et al. (2016), que expone que si la elevación de la lámina de agua supera la cota de la berma se produce transporte transversal de sedimentos, que altera la forma del perfil de playa. En la zona de estudio la altura de la berma es igual a $1.52 \mathrm{~m}$.

\subsection{Medidas}

\subsubsection{Hidrodinámicas}

Las medidas hidrodinámicas fueron realizadas en una campaña de campo de 39 días de duración (23 de diciembre de 2014 - 30 de enero de 2015) mediante dos perfiladores de corriente fondeados en la zona de estudio (Figura 1).

\subsubsection{Batimetrías}

Al inicio del periodo de estudio se realizó una batimetría completa de la zona deltaica, desde el Peñón de Salobreña hasta el Puerto de Motril. Las medidas fueron realizadas con una sonda multihaz GeoSwath Plus y una estación GPS diferencial GPS Trimble 5700. Los datos obtenidos fueron referenciados al elipsoide WGS84 y al cero hidrográfico del Puerto de Motril. Esta batimetría completa se usó para aplicar el modelo Delft3D de propagación del oleaje.

Posteriormente, se realizaron dos mediciones batimétricas más. Una a mitad del periodo de estudio (23 de marzo de 2015) y otra al final (21 de junio de 2015). Estas no cubrieron la totalidad de la zona deltaica, sino que se centraron en dos áreas de estudio específicas. La primera frente a la zona regenerada (P1) y la segunda en la zona intermedia entre la desembocadura del río y la Punta del Santo (P2). Esta segunda fue elegida debido a que se ha comprobado que es representativa del comportamiento general del tramo de costa entre la desembocadura y la Punta del Santo (Bergillos et al., 2016). En cada una de estas zonas, se realizaron 5 perfiles batimétricos separados $5 \mathrm{~m}$ cada uno. Con estos datos, se obtuvo un único perfil promediado para cada zona y en cada campaña, reduciendo así la incertidumbre asociada a los posibles errores de medida y a la variabilidad longitudinal del perfil. Con estas medidas batimétricas específicas se obtuvo la variación del volumen de sedimentos por metro de línea de costa en cada una de las áreas estudiadas entre cada campaña batimétrica. Posteriormente, esta variación de volumen se utilizó para tantear y comprobar el ajuste de los volúmenes de transporte longitudinal de sedimentos calculados mediante las distintas formulaciones.

\subsubsection{Topografía}

Durante el periodo de estudio se realizaron 5 medidas topográficas de la posición de la línea de costa en el tramo afectado por la regeneración (Tabla 1). Cada una de las medidas fueron hechas durante la bajamar, mediante un GPS diferencial Javad Maxor con menos de $2 \mathrm{~cm}$ de error tanto horizontal como verticalmente. Estos datos fueron usados para estudiar la evolución de la línea de 
costa. Además, también se utilizaron para comparar la línea de costa obtenida mediante el modelo de una línea y obtener así la bondad del ajuste de este para cada una de las distintas formulaciones.

\begin{tabular}{cccccc}
\hline $\mathrm{N}^{\mathrm{o}}$ Campaña & 1 & 2 & 3 & 4 & 5 \\
\hline Fecha & $23 / 12$ & $06 / 02$ & $23 / 03$ & $07 / 05$ & $21 / 06$ \\
Tipo de medida & $\mathrm{B}+\mathrm{T}$ & $\mathrm{T}$ & $\mathrm{B}+\mathrm{T}$ & $\mathrm{T}$ & $\mathrm{B}+\mathrm{T}$ \\
\hline
\end{tabular}

Tabla 1: Fechas en que se realizaron las campañas batimétricas (B) y topográficas $(\mathrm{T})$ durante el periodo de estudio.

\subsection{Modelos}

\subsubsection{Propagación de oleaje}

Los datos del punto SIMAR 2041080 proporcionados por Puertos de Estado fueron propagados desde aguas profundas hasta la costa usando el módulo Wave del modelo numérico Delft3D (Lesser et al., 2004; Lesser, 2009), basado en el modelo SWAN (Holthuijsen et al., 1993). La utilización de este modelo viene motivada por la experiencia que el grupo de Dinámica de Flujos Ambientales acumula en el uso de éste en playas como Carchuna (Ortega-Sánchez et al., 2014). Dichos datos se han usado para obtener las características del oleaje en rotura que son necesarios para las distintas formulaciones de transporte de sedimentos.

Para el modelo se usaron dos mallas distintas (Figura 1). Una malla gruesa, curvilínea de $82 \times 82$ celdas que cubre el total de la zona de estudio. El tamaño de celda decrece con la profundidad desde 170x65 hasta 80x80 $\mathrm{m}$. La segunda es una malla anidada, que cubre la zona más cercana a la costa desde el Peñón hasta el Puerto de Motril. Tiene un tamaño de 244x82 celdas de aproximadamente $25 \times 20$ m cada una. Para la resolución espectral del modelo se han usado 37 frecuencias logarítmicamente distribuidas que cubren desde 0.03 hasta $1 \mathrm{~Hz}$, mientras que para el espacio direccional, se han usado 72 direcciones que cubren $\operatorname{los} 360^{\circ}$ cada $5^{\circ}$.

El modelo fue calibrado por Bergillos et al. (2015) comparando los datos de altura de ola y dirección medidos en la campaña de campo descrita en la sección 3.2.1 con los resultados del modelo, obteniéndose coeficientes de determinación $\left(R^{2}\right)$ iguales a 0.8676 (A1) y 0.8894 (A2) (Figura 4). Los datos de entrada del modelo fueron los obtenidos del punto SIMAR, usando la batimetría inicial (Sección 3.2.2). Se consideraron los siguientes procesos físicos: viento, refracción, white-capping, rotura inducida por fondo $(\alpha=1$, $\gamma=0,73)$, interacciones triádicas no lineales $(\alpha=0,1$, $\beta=2,2$ ), rozamiento con el fondo (Tipo Collins, coeficiente $=0.02$ ) y difracción (smoothing coefficient $=0.6$, smoothing steps $=600$ ).

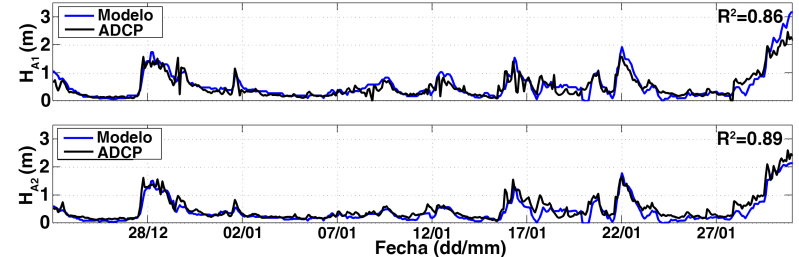

Figura 4: Calibración del modelo Delft3D Wave a partir de las medidas hidrodinámicas y coeficiente de correlación $R^{2}$.

Los resultados del modelo, además de ser utilizados como entrada de las distintas formulaciones de transporte, se usaron para calcular la distribución espacial del flujo medio de energía del oleaje, obtenido como:

$$
F=\rho g^{2} H_{0}^{2} \frac{T}{32 \pi}
$$

Se calculó el flujo de energía para cada estado de mar, y se realizó la media (temporal) de éste para cada uno de los periodos considerados.

\subsubsection{Transporte longitudinal de sedimentos}

Con el fin de obtener la redistribución de los sedimentos regenerados y los cambios producidos en la línea de costa durante el periodo de estudio, se usaron cuatro formulas diferentes considerando los tres tamaños de sedimento predominantes en la playa $(0.35 \mathrm{~mm}, 5 \mathrm{~mm}$ y 2 $\mathrm{cm})$ así como el tamaño usado para la regeneración (2 $\mathrm{mm}$ ). Las formulaciones se aplicaron teniendo en cuenta las variaciones longitudinales en la línea de costa, las características del oleaje en rotura y la pendiente de la zona de rompientes, para lo que se definieron un total de 341 perfiles de playa (perpendiculares a la línea de costa) espaciados 20 m entre el peñón de Salobreña y el puerto de Motril.

Para la obtención de estos datos a lo largo de la costa, se partió de los resultados obtenidos en el modelo numérico Delft3D. Puesto que los resultados del modelo se presentan para cada una de las celdas de la malla, las cuales no siempre se encuentran distribuidas perpendicularmente a la línea de costa, se interpolaron los resultados obtenidos por el Delft3D (4610 matrices correspondientes a cada estado de mar por cada una de las siguientes variables: altura de ola, dirección, periodo y fraction breaking) en los 341 perfiles. El fraction breaking representa el porcentaje de olas que rompen en cada celda (Battjes and Janssen, 1978) y fue utilizado para obtener los datos en rotura. Siguiendo el criterio de Van Rijn (2014) se tomó como punto de rotura aquel en el que rompe el $5 \%$ de las olas. 
Las formulaciones usadas para el cálculo de las tasas de transporte longitudinal de sedimentos fueron las siguientes. En primer lugar, la fórmula del CERC (USACE, 1984), por ser la más usada históricamente en la práctica de la ingeniería de costas, y por haber sido previamente testada en la zona de estudio por Ávila (2007) en condiciones naturales. Se basa en que la tasa de transporte longitudinal de sedimentos $(Q)$ es proporcional a la energía del oleaje por unidad de longitud de la costa y se expresa de la siguiente forma:

$$
Q=\frac{K \rho \sqrt{g}}{16 \sqrt{\gamma}\left(\rho_{s}-\rho\right)(1-p)} H_{b}^{2,5} \sin 2 \theta_{b}
$$

donde $Q$ es la tasa de transporte longitudinal de sedimentos expresada en volumen por unidad de tiempo, $K$ es un coeficiente empírico, $\rho$ es la densidad del agua, $\rho_{s}$ es la densidad del sedimento, $g$ es la aceleración de la gravedad, $p$ es la porosidad ( $p=0,4), H_{b}$ es la altura de ola significante en rotura, $\gamma$ es el índice de rotura, que varía entre 0.5 y 0.8 y fue utilizado como índice de calibración, y $\theta_{b}$ es el ángulo que forma el frente de oleaje con la línea de costa en la profundidad de rotura.

USACE (1984) recomienda un valor de $K=0,39$ derivado del estudio original realizado por Komar and Inman (1970). Sin embargo, Del Valle et al. (1993) encontraron la siguiente relación exponencial para el coeficiente $K$ y el tamaño del sedimento $D_{50}$ :

$$
K=1,6 e^{-2,5 D_{50}}
$$

En un primer tanteo, se hizo uso de esta expresión para cada una de los tamaños de grano considerados. Sin embargo, para las fracciones de grava se obtuvieron tasas de transporte del orden de $10^{-22}$. Por ello, se decidió adoptar para dichas fracciones el mínimo $K$ calibrado por Del Valle et al. (1993), a través de la comparación con medidas de campo, en una zona próxima a Playa Granada (Adra, Almería): 0.03 para $D \sim 2 \mathrm{~mm}$.

Inman and Bagnold (1963) desarrolló una formulación basada en que la energía del oleaje es invertida en levantar y transportar el sedimento del fondo. La expresión correspondiente es:

$$
Q=\frac{\sqrt{2}}{8} \frac{K}{\left(\rho_{s}-\rho\right)(1-p)} \rho \sqrt{g} H_{b}^{2,5} \sin 2 \theta_{b}
$$

Ésta ha sido utilizada en playas cercanas a nuestra zona de estudio, como la de Carchuna (Ortega-Sánchez et al., 2014), obteniendo resultados consistentes.

Por su parte, Kamphuis (1991) estudió la influencia de la pendiente de la zona de rompientes y el tamaño de grano en la tasa de transporte de sedimentos, siendo ambos factores importantes en la zona de estudio por su escarpada morfología (Jabaloy-Sánchez et al., 2014) y su variabilidad de sedimentos (Bárcenas et al., 2011). De su estudio derivó la siguiente formulación:

$$
Q_{m}=2,27 H_{b}^{2} T_{p}^{1,5} \tan (\beta)^{0,75} D_{50}^{-0,25} \sin 2 \theta_{b}
$$

donde $Q_{m}$ es la tasa de transporte de masa sumergida por unidad de tiempo y $\tan (\beta)$ es la pendiente en la zona de rompientes. El peso sumergido se relaciona con la tasa volumétrica de la siguiente forma $Q_{m}=\left(\rho_{s}-\rho\right)(1-$ p) $Q$.

Por último, debido a la mencionada variabilidad del tamaño de sedimento en la zona de estudio, se aplicó la fórmula propuesta por Van Rijn (2014), deducida tanto para arenas como para gravas. La tasa de transporte dada por esta formulación se obtiene como:

$$
Q_{m}=0,00018 K_{V R} \rho_{s} g^{0,5} \tan (\beta)^{0,4} D_{50}^{-0,6} H_{b}^{3,1} \sin 2 \theta_{b}
$$

donde $K_{V R}$ es un coeficiente que depende del tipo de oleaje y que varía entre 1 y 1,5. Esta expresión ha sido probada en playas heterogéneas sujetas a bidireccionalidad del oleaje (condiciones similares a las de Playa Granada) en el este del Reino Unido, obteniéndose resultados consistentes (French and Burningham, 2015).

Para medir la validez de las distintas formulaciones en la zona de estudio se obtuvo el coeficiente $C=$ $V_{\text {mod }} / V_{\text {med }}$, donde $V_{\text {med }}$ es el cambio de volumen observado en los perfiles obtenidos en las medidas batimétricas (sección 3.2.2) y $V_{\text {mod }}$ es el volumen acumulado calculado mediante las distintas formulaciones a través de la siguiente ecuación:

$$
V=\sum_{1}^{N} \frac{\left(Q_{i, n}-Q_{i+1, n}\right) \Delta t}{\Delta x}
$$

siendo $\mathrm{N}$ el número de estados de mar en el periodo considerado, $\mathrm{Q}_{i}$ la tasa de transporte en el perfil i, $\Delta \mathrm{t}$ la duración del estado de mar y $\Delta \mathrm{x}$ la separación espacial entre los dos perfiles

Ademas se estimó la desviación total con respecto al valor observado mediante la siguiente expresión:

$$
\epsilon=\sum|1-C|
$$

\subsubsection{Modelo de una línea}

A partir de las tasas de transporte calculadas para las cuatros formulaciones, se aplicó el modelo de una línea 

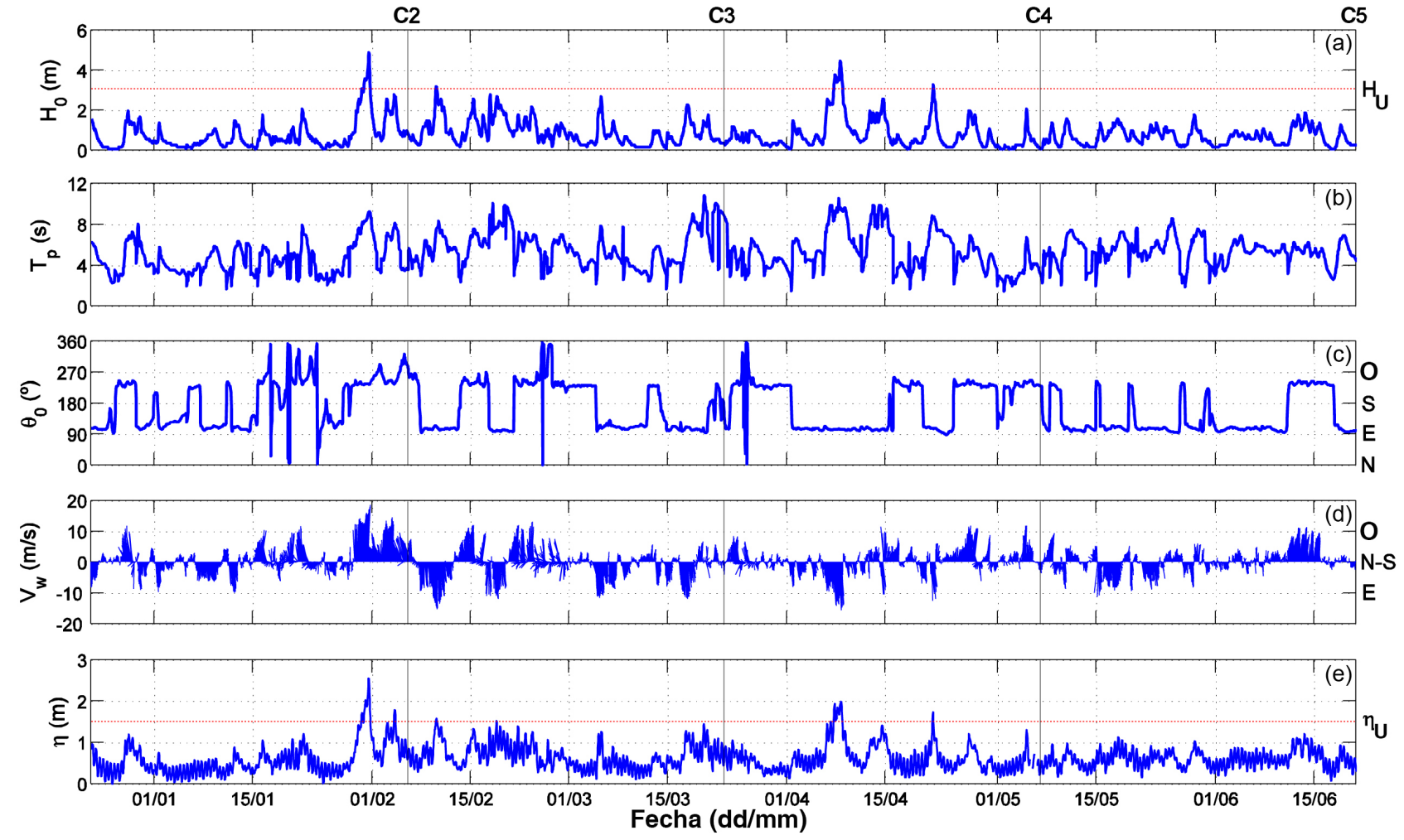

Figura 5: Clima marítimo durante el periodo de estudio. (a) Altura de ola significante. (b) Periodo de pico. (c) Dirección de procedencia del oleaje. (d) Módulo de la velocidad del viento. (e) Elevación total de la superficie libre.

entre cada una de las medidas topográficas realizadas. La expresión del modelo es:

$$
\frac{\partial y_{s}}{\partial t}=\frac{1}{D}\left(-\frac{\partial Q}{\partial x}\right)
$$

siendo $D=D_{C}+D_{B}$ donde $D_{C}$ es la profundidad de cierre y $D_{B}$ es la altura de la berma. Para cuantificar el error producido por el modelo con respecto a las observaciones topográficas realizadas en las distintas campañas de campo se calculó el error de la raiz cuadrada de la media (RMSE) a partir de la siguiente expresión:

$$
R M S E=\sqrt{\frac{\sum_{t=1}^{N}\left(\hat{y}_{t}-y_{t}\right)^{2}}{N}}
$$

donde $\hat{y}_{t}$ representa la posición de la línea de costa calculada por el modelo y $y_{t}$ la medida realmente.

\section{Resultados}

\subsection{Clima marítimo}

La figura 5 representa la altura de ola, el periodo pico, la dirección de procedencia del oleaje, el módulo de la velocidad del viento y la elevación total de la superfie libre durante el periodo de estudio. Considerando como evento de tormenta aquellos instantes en los que la altura de ola en profundidades indefinidas supera los $3.1 \mathrm{~m}$, en este caso se comprueba que durante el primer periodo existe un evento, en el segundo periodo un evento, en el tercer periodo dos y en el cuarto periodo ya no encontramos ningún evento de tormenta (Figura 5a). De todos ellos, el de mayor magnitud es el que encontramos en el primer periodo, con una duración de 22 horas y una altura de ola significante máxima de $4.9 \mathrm{~m}$. Durante todo el periodo de estudio, el periodo de pico osciló entre los 3 y los 10 s (Figura 5b).

En cuanto a la dirección de procedencia, durante el periodo de estudio se alternaron temporales procedentes del este y del oeste. Durante el evento de enero de 2015, la dirección de procedencia fue el oeste (Figura 5c). Sin embargo, en el de febrero y en los dos de abril el oleaje provenía de la dirección este. Por otra parte, la máxima velocidad del viento durante todo el periodo fue de 19.9 $\mathrm{m} / \mathrm{s}$ coincidiendo con el primer temporal, siendo menor a $10 \mathrm{~m} / \mathrm{s}$ el $90 \%$ del tiempo (Fig 5d).

El análisis de los datos de elevación de la superficie 
libre y run-up (Figura 5e) nos indica que los eventos erosivos en la zona de estudio no coinciden siempre con los temporales identificados con la altura de ola significante. Rebasándose la cota de la berma dos veces en el periodo $\mathrm{C} 1-\mathrm{C} 2$, dos en el C2-C3, dos en el C3-C4 y ninguna en el C4-C5.
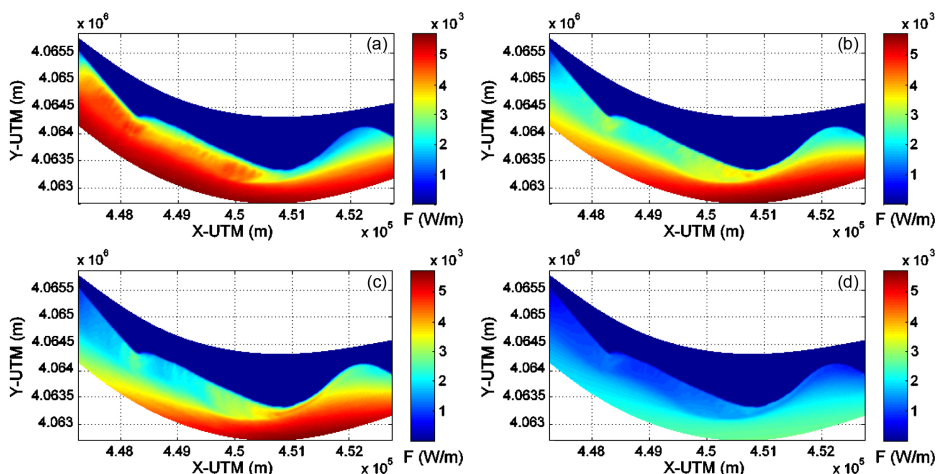

Figura 6: Distribución en planta del flujo de energía medio entre medidas topográficas.

\subsection{Propagación del oleaje}

La mayoría de las formulaciones para el cálculo de las tasas de transporte de sedimentos se basan en el flujo de energía del oleaje. En la figura 6 se representa el flujo de energía medio en cada periodo. Se observa que los mayores contenidos energéticos en Playa Granada se tienen en el primer periodo. Por su parte en los periodos 2, 3 y 4 el mayor flujo de energía se encuentra en la Punta del Santo. Esto se debe a que, como ya se expuso en el apartado 4.1, en el primer periodo se produjo un temporal procedente del suroeste, sin embargo los temporales que encontramos en el segundo y tercer periodo provienen del este. La morfología del tramo de costa hace que la Punta del Santo actúe como una barrera natural en temporales del este-sureste, haciendo que el flujo de energía que llega a Playa Granada sea mucho menor en este caso que cuando el temporal proviene del oeste-suroeste. Por otra parte, el flujo de energía medio en el cuarto periodo es sensiblemente menor que en los tres anteriores, lo que concuerda con los datos de clima marítimo en profundidades indefinidas, ya que en el cuarto periodo no se produjo ningún temporal.

\subsection{Evolución del perfil de playa}

Comparando las distintas medidas batimétricas realizadas se obtuvieron los cambios en el volumen por metro de línea de costa. Este volumen unitario se calculó en primer lugar para la totalidad del perfil hasta la profundidad de $10 \mathrm{~m}$ y por otro en la zona de rompientes.
Como límite para esta última se fijó la máxima profundidad de rotura alcanzada para los dos perfiles observa$\operatorname{dos}(5 \mathrm{~m})$.
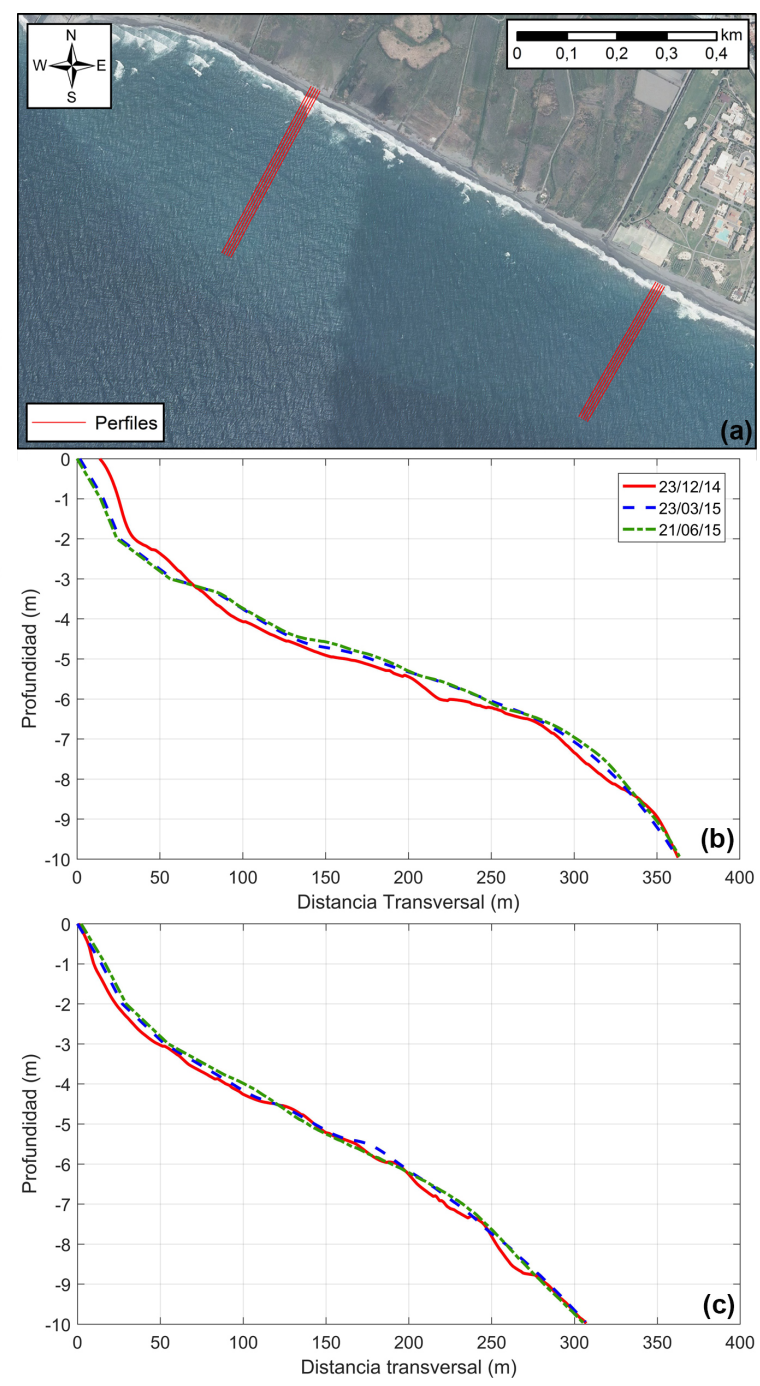

Figura 7: (a) Perfiles medidos en las campañas batimétricas. Evolución de los perfiles promediados, (b) P1 y (c) P2.

En el perfil P1, durante el periodo entre las campañas 1 y 3 se observa una importante pérdida de volumen hasta la batimétrica $3 \mathrm{~m}$ (Figura 7b). Sin embargo, el volumen aumenta a partir de dicha profundidad. Esto parece indicar que durante este periodo se ha producido un transporte transversal del sedimento regenerado. Al realizar la regeneración, el perfil se aleja de su forma de equilibrio, que ante la acción del oleaje tiende a recuperarse. El balance en el volumen de sedimentos en el total del perfil es de $17.96 \mathrm{~m}^{3} / \mathrm{m}$ lo que parece indicar que también se produce transporte longitudinal a la línea de 
costa de parte del sedimento erosionado. En el periodo entre las campañas 3 y 5 son menores los cambios en la forma del perfil. Esto puede responder, por una parte al menor flujo energético del oleaje durante este periodo (Sección 4.2) y por otra parte a la mayor similitud de la forma del perfil con el que se tenía en condiciones naturales, lo que limita el transporte transversal. En este caso, el balance en el volumen es de $13.06 \mathrm{~m}^{3} / \mathrm{m}$, que de nuevo refleja la importancia del transporte longitudinal del sedimento erosionado.

En el perfil P2, durante el periodo entre las campañas 1 y 3 , se produjo una ganancia de $38.66 \mathrm{~m}^{3} / \mathrm{m}$ con una distribución transversal mucho más uniforme que en el perfil P1 (Figura 7c). Esta ganancia de volumen en el perfil P2 parece indicar que parte del sedimento erosionado en los alrededores de la desembocadura del río se ha sido transportado hasta esta zona debido a los temporales con oleaje proveniente del suroeste que tuvieron lugar en este periodo (Sección 4.1). Durante el periodo entre las campañas 3 y 5 este balance de volumen desciende hasta $3.65 \mathrm{~m}^{3} / \mathrm{m}$. En cuanto a los balances de volumen en la zona de rompientes, en el periodo entre las campañas 1 y 3 en el perfil P1 es de $-12.59 \mathrm{~m}^{3} / \mathrm{m}$ mientras que en el perfil P2 es $17.14 \mathrm{~m}^{3} / \mathrm{m}$. Por su parte, en el periodo entre las campañas 3 y 5 en el perfil $\mathrm{P} 1$ es de $0.6 \mathrm{~m}^{3} / \mathrm{m}$ y en el perfil P2 es $12.3 \mathrm{~m}^{3} / \mathrm{m}$. Estos datos confirman la importancia del transporte longitudinal de sedimento entre estos dos perfiles.

\subsection{Evolución de la línea de costa}

Durante el periodo entre las campañas 1 y 2 tuvo lugar una pérdida de área de playa de $9366 \mathrm{~m}^{2}$ (Figura $8 b)$. Esta erosión puede responder, por un lado, al fuerte temporal de enero de 2015, con oleajes en dirección oeste-suroeste que inciden directamente en este tramo de costa, transportando sedimento hacia el este. Por otro lado, el desvío que la regeneración produce en la línea de costa con respecto a su forma en condiciones naturales (Figura 8a), también ha podido acelerar la erosión. Durante los periodos entre las campañas 2-3 y 3-4 la línea de costa avanzó, incrementando el área de la playa en 8864 y $4203 \mathrm{~m}^{2}$ respectivamente. Estos resultados responden a que los temporales producidos durante estos dos periodos provenían de la dirección este-sureste, provocando que el sedimento sea transportado hasta esta zona. Por último, la variación del área de playa en el periodo entre las campañas 4 y 5 es el menor de todos con $-3513 \mathrm{~m}^{2}$, lo que se corresponde con que este periodo es el de menor contenido energético del oleaje de los 4 estudiados.
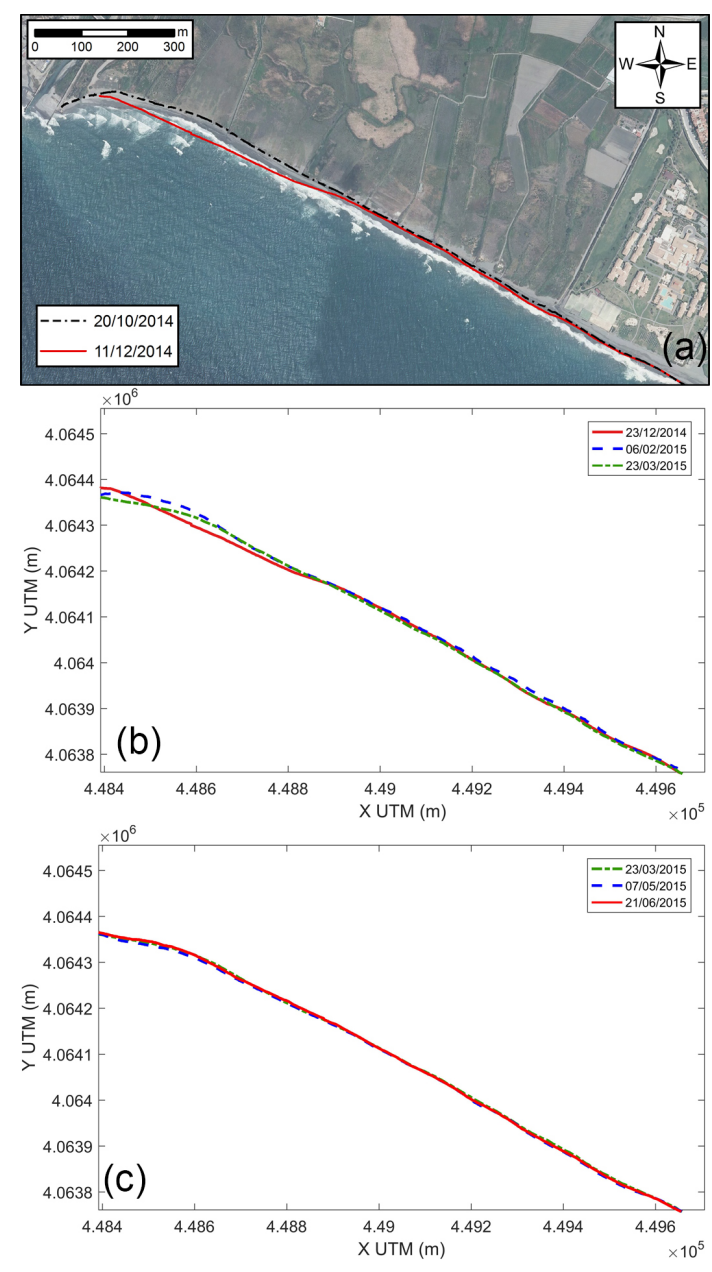

Figura 8: (a) Línea de costa antes y después de la regeneración. (b) y (c) Evolución de la línea de costa durante el periodo de estudio.

\subsection{Transporte longitudinal de sedimentos: compara- ción y discusión}

Los resultados obtenidos son muy variables dependiendo de la formulación y el tamaño de sedimento considerado (Tabla 2). El mejor ajuste se obtiene usando la fórmula de Van Rijn (2014) con $\mathrm{D}_{50}=2 \mathrm{~cm}$, obteniendo un coeficiente $\mathrm{C}$ de 1.01 y 0.97 para los perfiles P1 y P2 respectivamente en el periodo 1-3, y 2.66 y 0.39 en el periodo 3-5 (Tabla 2), lo que supone un $\epsilon$ total de 2.41. En general, para esta formulación cuanto menor es el $\mathrm{D}_{50}$ considerado peor es el ajuste, siendo $\epsilon=7.08, \epsilon=14.2$ y $\epsilon=87.42$ para $\mathrm{D}_{50}=5 \mathrm{~mm}, \mathrm{D}_{50}=2 \mathrm{~mm}$ y $\mathrm{D}_{50}=0.35 \mathrm{~mm}$ respectivamente. Estos resultados concuerdan con los obtenidos por French and Burningham (2015), que ya mostraba un buen ajuste de esta formulación para playas heterogéneas sometidas a oleajes bidireccionales y con incidencia oblicua. 

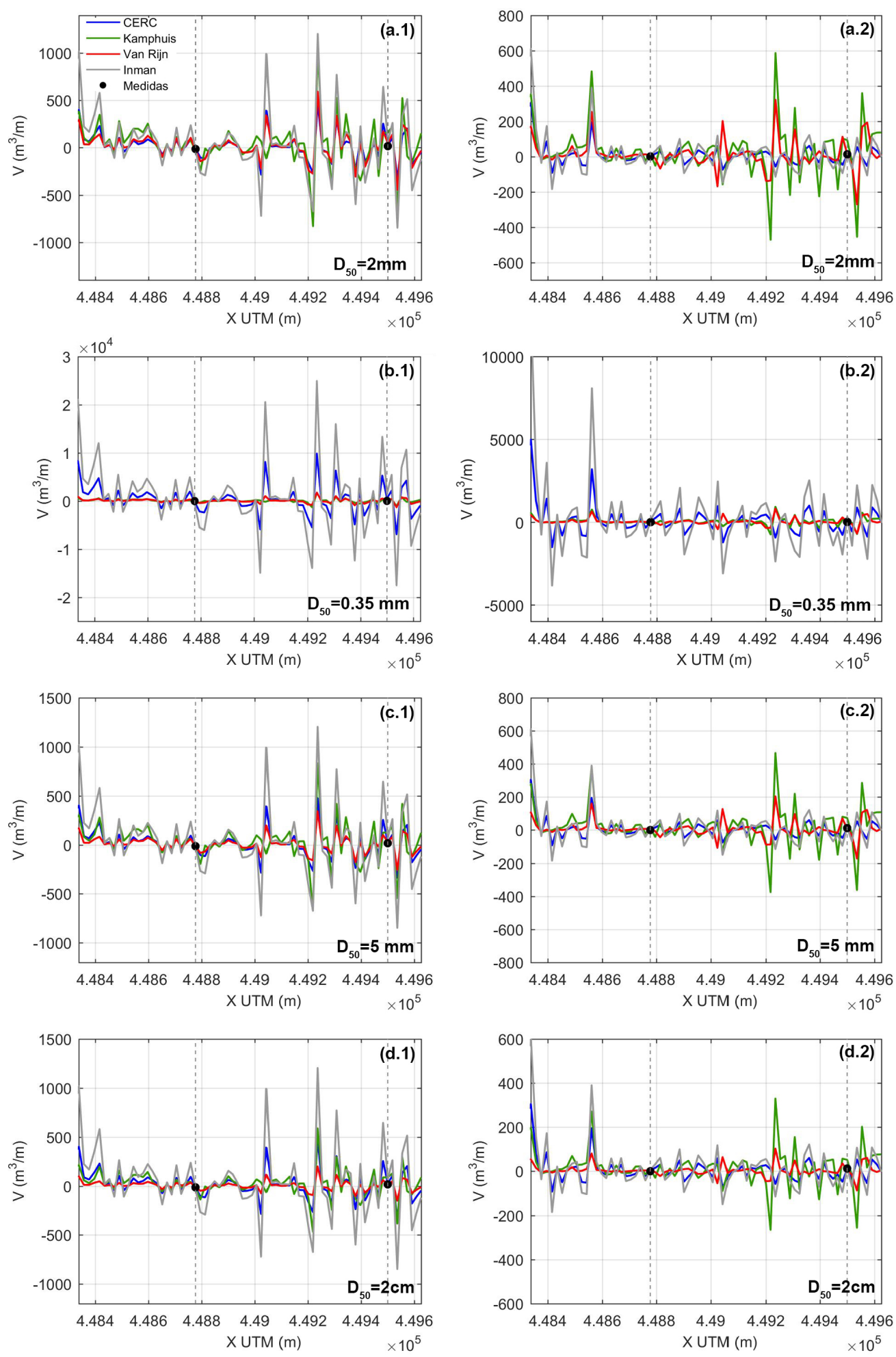

Figura 9: Volumen unitario de sedimentos acumulado para cada fracción de tamaño de grano (a), (b), (c) y (d) y para cada periodo (1) y (2). 
En el caso de la fórmula del CERC, los resultados para todas las fracciones de grava son los mismo debido a las limitaciones del coeficiente $\mathrm{K}$. El ajuste para la fracción de grava arroja un $\epsilon$ de 5.68 con C entre 0.65 y 5.47 , mientras que para la fracción de arena el error se eleva hasta 144.23 , llegando a obtener un $\mathrm{C}=89.48$ en el primer perfil para el periodo 1-3.

$\mathrm{Al}$ igual que la expresión anterior, la propuesta por Inman and Bagnold (1963) solo distingue entre arena y gravas, ya que también hace uso del coeficiente $\mathrm{K}$. Los resultados son similares a los anteriores. Tan solo se observa una tendencia a sobrestimar el transporte en Inman and Bagnold (1963) mayor que en la del CERC. En este caso, $\epsilon$ es igual a 14.1 para las gravas, y en arenas se eleva hasta 371.

Por último, la ecuación de Kamphuis (1991) sobrestima el transporte en todas las fracciones obteniendo C $>1$ en todos los casos estudiados. Esta sobrestimación es menor en el caso de la fracción $\mathrm{D}_{50}=2 \mathrm{~cm}$, con C entre 1.29 y 18.03, lo que supone un $\epsilon$ de 24.74. Esta tendencia a sobrestimar las tasas de transporte en playas de grava por parte de la formulación de Kamphuis (1991) ya fue expuesta por Reeve et al. (2004).

La que mejor ajusta en todas las formulaciones es $\mathrm{D}_{50}=2 \mathrm{~cm}$, con $\epsilon$ entre 2.41 y 24 ; mientras que la que peor ajuste obtiene es $\mathrm{D}_{50}=0.35 \mathrm{~mm}$, con $\epsilon$ entre $76.5 \mathrm{y}$ 371. Las fracciones $\mathrm{D}_{50}=5 \mathrm{~mm}$ y $\mathrm{D}_{50}=2 \mathrm{~mm}$ ofrecen un ajuste más cercano al de $\mathrm{D}_{50}=2 \mathrm{~cm}$ que al de la fracción de arena $\left(D_{50}=0.35 \mathrm{~mm}\right)$. Parece, por tanto, que en playas heterogéneas las fracciones más gruesas tienen una influencia mayor en la respuesta y forma de la playa que las fracciones más finas.

\begin{tabular}{cc|cccc}
\hline & & \multicolumn{2}{|c}{ Perfil 1 } & \multicolumn{2}{c}{ Perfil 2 } \\
& & C1-C3 & C3-C5 & C1-C3 & C3-C5 \\
\hline \multirow{3}{*}{ Modelado } & $D_{50}=2 \mathrm{~mm}$ & 0.73 & 5.47 & 1.59 & 0.65 \\
CERC & $D_{50}=0,35 \mathrm{~mm}$ & 15.09 & 89.48 & 32.97 & 10.7 \\
& $D_{50}=5 \mathrm{~mm}$ & 0.73 & 5.47 & 1.59 & 0.65 \\
& $D_{50}=20 \mathrm{~mm}$ & 0.73 & 5.47 & 1.59 & 0.65 \\
\hline \multirow{3}{*}{ Modelado } & $D_{50}=2 \mathrm{~mm}$ & 1.84 & 10.93 & 4.03 & 1.31 \\
Inman & $D_{50}=0,35 \mathrm{~mm}$ & 38.18 & 226.36 & 83.4 & 27.06 \\
& $D_{50}=5 \mathrm{~mm}$ & 1.84 & 10.93 & 4.03 & 1.31 \\
& $D_{50}=20 \mathrm{~mm}$ & 1.84 & 10.93 & 4.03 & 1.31 \\
\hline \multirow{3}{*}{ Modelado } & $D_{50}=2 \mathrm{~mm}$ & 2.29 & 32.06 & 9.2 & 7.56 \\
Kamphuis & $D_{50}=0,35 \mathrm{~mm}$ & 3.6 & 14.49 & 50.5 & 11.91 \\
& $D_{50}=5 \mathrm{~mm}$ & 1.82 & 25.5 & 7.32 & 6.01 \\
& $D_{50}=20 \mathrm{~mm}$ & 1.29 & 8.03 & 5.17 & 4.25 \\
\hline \multirow{3}{*}{ Modelado } & $D_{50}=2 \mathrm{~mm}$ & 2.37 & 10.77 & 3.89 & 1.15 \\
Van Rijn & $D_{50}=0,35 \mathrm{~mm}$ & 7.06 & 69.4 & 11.57 & 3.39 \\
& $D_{50}=5 \mathrm{~mm}$ & 1.37 & 6.12 & 2.24 & 0.66 \\
& $D_{50}=20 \mathrm{~mm}$ & 1.01 & 2.66 & 0.98 & 0.39 \\
\hline
\end{tabular}

Tabla 2: Coeficiente $C=V_{\text {mod }} / V_{o b s}$.

Atendiendo al volumen acumulado en el total del área regenerada 9) se observa que en el primer periodo, Inman and Bagnold (1963) es el que mayor tasa de transporte obtiene (Figura 9a.1, b.1, c.1 y d.1). También se comprueba que la expresión de Kamphuis (1991) obtiene tasas mayores al resto en las fracciones de grava, sobre todo en la parte más oriental de la regeneración. Sin embargo, tomando la fracción de arena $\left(D_{50}=0.35\right.$ $\mathrm{mm}$ ) la que mayor tasa de transporte obtiene junto con Inman and Bagnold (1963) es la fórmula del CERC. Por último, Van Rijn (2014) obtiene menores tasas de transporte que el resto para todas las fracciones.

\subsection{Modelo de una línea: comparación y discusión}

Los resultados del modelo de una línea para cada una de las tasas de transporte calculadas con las distintas formulaciones se observan en la figura 10. De nuevo, la formulación que mejor modela la evolución de la línea de costa en la zona de estudio es la de Van Rijn (2014), con un $R M S E_{\text {total }}=35.84 \mathrm{~m}$ (Tabla 4.6). Las formulaciones de Kamphuis (1991) y el CERC proporcionan un $R M S E_{\text {total }}$ de $46.98 \mathrm{~m}$ y $48.19 \mathrm{~m}$, respectivamente. Por último, la que mayor error obtiene en su predicción es la expresión de Inman and Bagnold (1963), con 83.66 $\mathrm{m}$.

En cuanto a los errores obtenidos en cada periodo entre campañas, se observa que estos descienden con el tiempo. Para el primer periodo, justo después de la regeneración, el error oscila entre 17.65 m y 19.07 m para las tres formulaciones que mejores resultados obtienen. Sin embargo, en el último periodo, el error oscila entre $3.5 \mathrm{~m}$ y $5 \mathrm{~m}$. Esto podría indicar que el modelo de una línea, considerando la expresión de Van Rijn (2014), es capaz de ajustar mejor la posición de la línea de costa cuanto más cercanas sean las condiciones a las naturales y que sea la influencia de la regeneración en la forma del perfil de playa lo que nos induce los mayores errores en los dos primeros periodos.

\begin{tabular}{l|llll} 
RMSE & CERC & Kamphuis & Van Rijn & Inman \\
\hline C1-C2 & 19.07 & 18.06 & 17.65 & 26.32 \\
C2-C3 & 14.38 & 15.6 & 10.12 & 31.15 \\
C3-C4 & 9.73 & 8.9 & 4.57 & 18.27 \\
C4-C5 & 5 & 4.42 & 3.5 & 7.93 \\
\hline RMS $E_{\text {total }}$ & 48.19 & 46.98 & 35.84 & 83.66
\end{tabular}

Tabla 3: RMSE de la posición de la línea de costa predicha por el modelo one line. 


\section{Conclusiones}

La morfología de Playa Granada, así como la bidireccionalidad en el oleaje de la zona caracterizan el comportamiento de la costa. El estudio del clima marítimo y de la propagación del oleaje nos indican que en Playa Granada el comportamiento es muy distinto para temporales que provienen de la dirección oeste-suroeste, los cuales alcanzan la costa con un contenido energético mayor, que para los que provienen de la dirección estesureste, para los cuales la Punta del Santo actúa como barrera natural disminuyendo su contenido energético (Figura 6).

El estudio de las medidas topográficas y batimétricas nos indica, por una parte, que la regeneración realizada aleja al perfil de playa de su posición de equilibrio, acelerando los procesos de erosión. Por otra parte, los temporales del oeste-suroeste parecen provocar un transporte longitudinal de sedimentos de oeste a este entre los perfiles batimétricos estudiados. Por el contrario, los temporales del este-sureste producen el transporte en sentido contrario, favoreciendo la recuperación de costa en Playa Granada.

La fórmula que mejor se ajusta a las medidas realizadas es la de Van Rijn (2014), usando como tamaño de grano la fracción dominante más gruesa. La fórmula del CERC es la que más se acerca a la de Van Rijn (2014), mientras que Kamphuis (1991) e Inman and Bagnold (1963) tienden a sobrestimar el transporte. El mejor ajuste en todas las formulaciones se obtiene con las fracciones más gruesas, aumentando el error conforme disminuimos el tamaño del sedimento.

Por su parte, el modelo de una línea se ve muy afectado por la presencia de la regeneración. El error en los dos primeros periodos entre campañas de campo es alto (17.65-19.07 m), comparado con el resto de periodos. Los resultados indican que dicho modelo describe mejor el comportamiento de la línea de costa cuanto más naturales sean las condiciones.

\section{Líneas de investigación futura}

Los resultados y conclusiones de este trabajo abren nuevas líneas de investigación que podrán ser desarrolladas en el futuro. En primer lugar, la combinación de las tasas de transporte de las distintas fracciones de tamaño de grano, obteniendo una expresión del tipo:

$$
Q=\alpha Q_{\text {arena }}+\beta Q_{\text {grava }}
$$

que permita modelar con mayor exactitud el transporte longitudinal en playas heterogéneas.
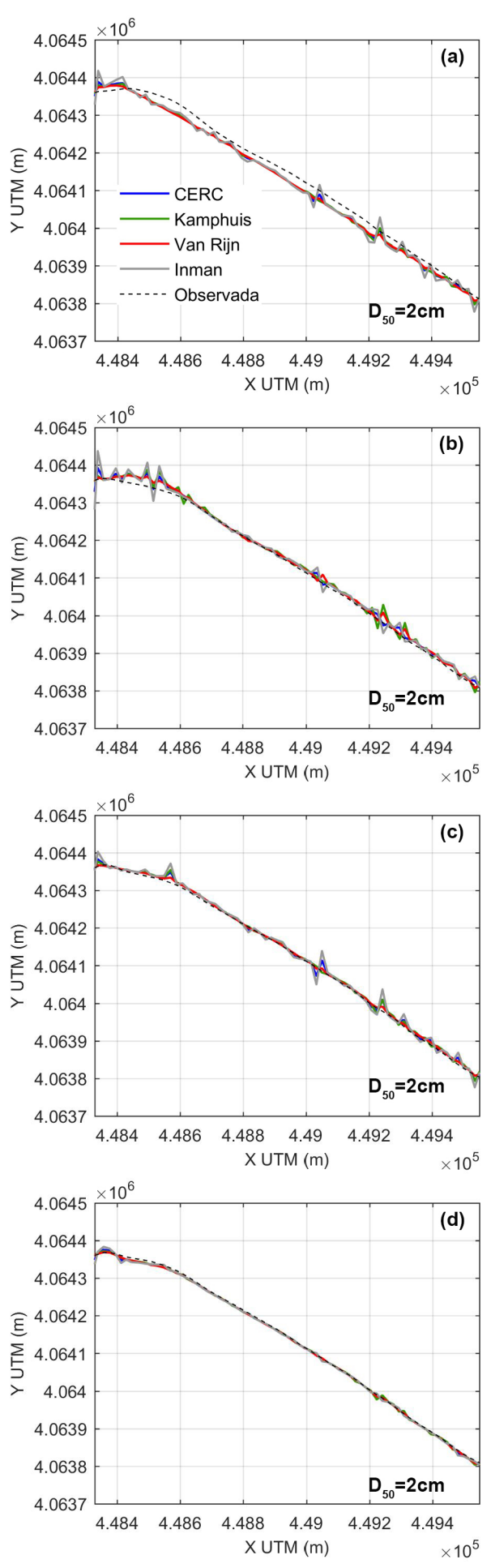

Figura 10: Línea de costa observada y la modelada durante cada periodo para el tamaño de sedimento que mejor ajuste proporciona. 
En segundo lugar, la comparación de los resultados obtenidos con los que se obtienen en la misma zona de estudio pero en condiciones naturales, con el fin de cuantificar la influencia de la regeneración en el comportamiento del sistema. Por último, se hace necesario la aplicación de un modelo de perfil de playa, como el XBeach-G (McCall et al., 2014, 2015) que compute el transporte transversal en combinación con los resultados ya obtenidos para el transporte longitudinal.

\section{Agradecimientos}

Quisiera dar las gracias a todos los que han contribuido a realizar este Trabajo Fin de Máster. En primer lugar a mis tutores: el Doctor Miguel Ortega Sánchez y Rafael Bergillos Meca. Sin su disponibilidad y consejos este trabajo no se podría haber llevado a cabo. En segundo lugar, al Grupo de Dinámica de Flujos Ambientales de la Universidad de Granada, por su acompañamiento, y en especial a Pedro Magaña, por el apoyo prestado. Por último, quisiera a agradecer a las Universidades de Granada, Córdoba y Málaga la organización del Máster Interuniversitario en Hidráulica Ambiental, en el que se inserta este trabajo.

\section{Referencias}

Ávila, A., 2007. Procesos de múltiple escala en la evolución de la línea de costa .

Bárcenas, P., Lobo, F., Macías, J., Fernández-Salas, L., del Río, V.D., 2011. Spatial variability of surficial sediments on the northern shelf of the Alboran Sea: the effects of hydrodynamic forcing and supply of sediment by rivers. Journal of Iberian Geology 37, 195-214.

Battjes, J., Janssen, J., 1978. Energy loss and set-up due to breaking of random waves. Coastal Engineering Proceedings 1.

Bergillos, R.J., Ortega-Sánchez, M., Losada, M.A., 2015. Foreshore evolution of a mixed sand and gravel beach: The case of Playa Granada (Southern Spain), in: Proceedings of the 8th Coastal Sediments, World Scientific.

Bergillos, R.J., Ortega-Sánchez, M., Masselink, G., Losada, M.A., 2016. Morpho-sedimentary dynamics of a micro-tidal mixed sand and gravel beach, Playa Granada, southern Spain. Marine Geology 379, 28-38.

Bertoni, D., Sarti, G., 2011. On the profile evolution of three artificial pebble beaches at Marina di Pisa, Italy. Geomorphology 130, $244-$ 254

Buscombe, D., Masselink, G., 2006. Concepts in gravel beach dynamics. Earth-Science Reviews 79, 33-52.

Del Valle, R., Medina, R., Losada, M.A., 1993. Dependence of coefficient $\mathrm{K}$ on grain size. Journal of waterway, port, coastal, and ocean engineering 119, 568-574.

El Banna, M.M., Frihy, O.E., 2009. Human-induced changes in the geomorphology of the northeastern coast of the nile delta, egypt. Geomorphology 107, 72-78.

Félix, A., Baquerizo, A., Santiago, J., Losada, M., 2012. Coastal zone management with stochastic multi-criteria analysis. Journal of environmental management 112, 252-266.
French, J., Burningham, H., 2015. Wave-driven sediment pathways on a gravel-dominated coast subject to a strongly bi-modal wave climate, suffolk, eastern uk, in: The Proceedings of the Coastal Sediments 2015. World Scientific.

Herrero, J., Polo, M.J., Losada, M.A., 2011. Snow evolution in sierra nevada (spain) from an energy balance model validated with landsat tm data, in: SPIE Remote Sensing, International Society for Optics and Photonics. pp. 817403-817403.

Holthuijsen, L., Booij, N., Ris, R., 1993. A spectral wave model for the coastal zone, in: Ocean Wave Measurement and Analysis (1993), ASCE. pp. 630-641.

Inman, D.L., Bagnold, R., 1963. Littoral processes. The sea 3, 529553.

IPCC, 2001. Climate change 2001: impacts, adaptation, and vulnerability: contribution of Working Group II to the third assessment report of the Intergovernmental Panel on Climate Change. Cambridge University Press.

Jabaloy-Sánchez, A., Lobo, F.J., Azor, A., Martín-Rosales, W., Pérez-Peña, J.V., Bárcenas, P., Macías, J., Fernández-Salas, L.M., Vázquez-Vílchez, M., 2014. Six thousand years of coastline evolution in the Guadalfeo deltaic system (southern Iberian Peninsula). Geomorphology 206, 374-391.

Jennings, R., Shulmeister, J., 2002. A field based classification scheme for gravel beaches. Marine Geology 186, 211-228.

Kamphuis, J.W., 1991. Alongshore sediment transport rate. Journal of Waterway, Port, Coastal, and Ocean Engineering 117, 624-640.

Komar, P.D., Inman, D.L., 1970. Longshore sand transport on beaches. Journal of geophysical research 75, 5914-5927.

Lesser, G., Roelvink, J., Van Kester, J., Stelling, G., 2004. Development and validation of a three-dimensional morphological model. Coastal engineering 51, 883-915.

Lesser, G.R., 2009. An approach to medium-term coastal morphological modeling. Ph.D. thesis. Department of Civil Engineering, Delft University of Technology, Delft, The Netherlands.

López-Ruiz, A., Ortega-Sánchez, M., Baquerizo, A., Losada, M.Á., 2014. A note on alongshore sediment transport on weakly curvilinear coasts and its implications. Coastal Engineering 88, 143-153.

Losada, M.A., Baquerizo, A., Ortega-Sánchez, M., Ávila, A., 2011. Coastal evolution, sea level, and assessment of intrinsic uncertainty. Journal of Coastal Research , 218-228.

McCall, R., Masselink, G., Poate, T., Roelvink, J., Almeida, L., 2015. Modelling the morphodynamics of gravel beaches during storms with xbeach-g. Coastal Engineering 103, 52-66.

McCall, R., Masselink, G., Poate, T., Roelvink, J., Almeida, L., Davidson, M., Russell, P., 2014. Modelling storm hydrodynamics on gravel beaches with XBeach-G. Coastal Engineering 91, 231-250.

Millares, A., Polo, M., Moñino, A., Herrero, J., Losada, M., 2014. Bedload dynamics and associated snowmelt influence in mountainous and semiarid alluvial rivers. Geomorphology 206, 330-342.

Moreno Aranda, I.M., Millares Valenzuela, A., Herrero Lantarón, J., Polo Gómez, M.J., Losada Rodríguez, M.Á., 2010. Futuro de los recursos hídricos en la cuenca del río guadalfeo bajo supuesto de cambio climático .

Nielsen, P., Hanslow, D.J., 1991. Wave runup distributions on natural beaches. Journal of Coastal Research , 1139-1152.

Ortega-Sánchez, M., Bramato, S., Quevedo, E., Mans, C., Losada, M.A., 2008. Atmospheric-hydrodynamic coupling in the nearshore. Geophysical Research Letters 35.

Ortega-Sánchez, M., Lobo, F., López-Ruiz, A., Losada, M., Fernández-Salas, L., 2014. The influence of shelf-indenting canyons and infralittoral prograding wedges on coastal morphology: The Carchuna system in Southern Spain. Marine Geology 347, 107-122.

Polo, M., Aguilar, C., Díaz-Gutiérrez, A., Dugo, M.G., 2013. Cuantificando la interceptación en cuencas heterogéneas: la cuenca del 
río guadalfeo (granada), in: Interceptación de la lluvia por la vegetación en España, Instituto Euromediterráneo del Agua. pp. 297318.

Reeve, D., Chadwick, A., Fleming, C., 2004. Coastal engineering: processes, theory and design practice. CRC Press.

Rodríguez-Delgado, C., 2015. Estudio de la evolución del delta del guadalfeo. Proyecto Fin de Carrera. Universidad de Granada .

de Schipper, M.A., de Vries, S., Ruessink, G., de Zeeuw, R.C., Rutten, J., van Gelder-Maas, C., Stive, M.J., 2016. Initial spreading of a mega feeder nourishment: Observations of the sand engine pilot project. Coastal Engineering 111, 23-38.

Simeoni, U., Corbau, C., 2009. A review of the delta po evolution (italy) related to climatic changes and human impacts. Geomorphology 107, 64-71.

Syvitski, J.P., Kettner, A.J., Overeem, I., Hutton, E.W., Hannon, M.T., Brakenridge, G.R., Day, J., Vörösmarty, C., Saito, Y., Giosan, L., et al., 2009. Sinking deltas due to human activities. Nature Geoscience 2, 681-686.

USACE, 1984. Shore Protection Manual. Coastal Engineering Research Center, Government Printing Office, Washington DC .

Van Rijn, L.C., 2014. A simple general expression for longshore transport of sand, gravel and shingle. Coastal Engineering 90, 23-39. 